\title{
Hearing loss and a supportive tactile signal in a navigation system: Effects on driving behavior and eye movements
}

\author{
Birgitta Thorslund \\ VTI (Swedish National Road and Transport Research Institute), Sweden
}

Björn Peters

VTI (Swedish National Road and Transport Research Institute), Sweden

Kenneth Holmqvist

University of Lund, Sweden

Alexander Black

School of Optometry, Queensland

University of Technology, Brisbane,
Nicholas Herbert

Institute for Transport Studies,

University of Leeds, UK

\author{
Björn Lidestam \\ Linnaeus Centre HEAD, University of \\ Linköping, Sweden \\ Björn Lyxell \\ Linnaeus Centre HEAD, University of \\ Linköping, Sweden
}

An on-road study was conducted to evaluate a complementary tactile navigation signal on driving behaviour and eye movements for drivers with hearing loss (HL) compared to drivers with normal hearing (NH). 32 participants (16 HL and $16 \mathrm{NH})$ performed two preprogrammed navigation tasks. In one, participants received only visual information, while the other also included a vibration in the seat to guide them in the correct direction. SMI glasses were used for eye tracking, recording the point of gaze within the scene. Analysis was performed on predefined regions. A questionnaire examined participant's experience of the navigation systems. Hearing loss was associated with lower speed, higher satisfaction with the tactile signal and more glances in the rear view mirror. Additionally, tactile support led to less time spent viewing the navigation display.

\section{Keywords: hearing loss, tactile support, navigation system, eye} movements, driving behavior

\section{Introduction}

The prevalence of hearing loss in Europe is roughly $30 \%$ for men and $20 \%$ for women at the age of 70 years, and $55 \%$ for men and $45 \%$ for women at the age of 80 years (Roth, 2001). A consequence of the increasing prevalence of hearing loss, is that the number of road users with hearing loss will also increase. The prevalence of age related hearing loss is increasing, due to populations becoming progressively older and thus presenting symptoms of reduced sensory function. The prevalence increases for all ages, although the most common category of hearing loss is presbycusis, which refers to the physiological age-related changes of the peripheral and central auditory system leading to hearing loss.

According to a questionnaire study, individuals with hearing loss drive to the same extent as normal hearing individuals (Thorslund, Peters, Lyxell, \& Lidestam, 2013). Some researchers have suggested that hearing loss is associated with higher risk of accidents (Barreto, Swerdlow, Smith, \& Higgins, 1997; Ivers, Mitchell, \& Cumming, 1999; Picard et al., 2008) while others have 
found no such relation (Green, 2013; McCloskey, Koepsell, Wolf, \& Buchner, 1994; Thorslund, Peters, Lyxell, et al., 2013) - though McCloskey et al. do find that hearing aid users are more at risk of accidents.

In a simulator study examining driving behavior related to hearing loss Thorslund et al. (2013) found that during a secondary task, participants with hearing loss looked more in the rear view mirror and had shorter but more frequent glances away from road compared to participants with normal hearing. This, in association with a lower speed, indicates a more cautious driving behavior possibly associated with coping strategies (Thorslund, Peters, Lidestam, \& Lyxell, 2013).

According to Fuller (2005) the level of difficulty in the driving task is associated with the spare capacity of driver capability and is connected to the cognitive workload. Among the factors determining driving task demand, speed is the one over which the driver has immediate control (Fuller, 2005). It has also been demonstrated that when a threshold of a certain preferred speed is exceeded, experienced task difficulty, effort and feeling of risk is affected (Lewis-Evans, 2011). This is interesting since it has been shown that differences in driving behavior associated with hearing loss appear either when driving complexity increases to a certain level, leading to speed reduction (Thorslund, Peters, Lidestam, et al., 2013) or when a secondary task is added or both in combination, leading to speed reduction (Thorslund, Peters, Lidestam, et al., 2013) and to a decrease in driving performance (Hickson, Wood, Chaparro, Lacherez, \& Marszalek, 2010). A decreased driving performance, in the presence of auditory or visual distractors, for participants with hearing loss compared to normal hearing participants has also been shown (Hickson, et al., 2010). Hearing loss has also been demonstrated to lead to a reduction in speeding violations (Picard, et al., 2008) and to decreased travelling speed in a driving simulator study (Thorslund, Peters, Lidestam, et al., 2013). Several studies have linked speed perception to the amount of noise in car cabins or to the driving sound (Evans, 1970; Ohta \& Komatsu, 1991). Thus, speed might be perceived differently by drivers with hearing loss, who typically have a reduced sensitivity to sounds.

There is a trend for more and more information and support systems being installed in cars which are used for navigation, distance or lane keeping, etc. These systems often use auditory output, which may make them less accessible for drivers with hearing loss. A tactile signal via a vibration in the seat, calling for driver attention, has been demonstrated as effective and highly accepted by drivers both with and without hearing loss (Thorslund, Peters, Lidestam, et al., 2013). This suggests tactile signaling as an appropriate or complementary modality when informing or supporting drivers. The purpose of the present study, conducted in real traffic, was to replicate and further examine driver coping strategies associated with hearing loss and evaluate the effect of additional tactile support in a navigation system. This tactile support was given as a vibration in either the left or the right side of the driving seat, to indicate the direction of the next turn. Eye movement patterns for drivers with and without hearing loss were compared, since a previous study indicates differences (Thorslund, Ahlstrom, et al., 2013).

\section{Methods}

\section{Design and expectations}

Knowing that a tactile signal is effective in calling for driver attention and has a high acceptability (Thorslund, Peters, Lidestam, et al., 2013), tactile support was expected to be associated with:

- more focus on the road

- better driving performance (less errors)

- $\quad$ higher satisfaction with the system

With previous research showing that slower driving speeds are associated with hearing loss (Picard, et al., 2008; Thorslund, Peters, Lidestam, et al., 2013), and indications of a more cautious driving behavior for drivers with hearing loss (Thorslund, Ahlstrom, et al., 2013), it was expected that participants with hearing loss would show a more cautious driving behavior compared to normally hearing participants. This cautious driving style is likely to be revealed by:

- $\quad$ a lower driving speed

- more glances in the mirrors

Since differences related to hearing loss have been shown to appear when a certain level of driving complexity is exceeded (Hickson, et al., 2010; Thorslund, Peters, Lidestam, et al., 2013) we expect to see more differences between the groups during the higher complexity condition. 
Journal of Eye Movement Research $6(5): 1,1-9$
Thorslund, B., Peters, B., Herbert, N., Holmqvist, K., Lidestam, B., Black, A., Lyxell, B. (2013) Hearing loss and a tactile signal in a navigation system: Driving behavior and eye movements
A $2 \times 2 \times 2$ split-plot factorial design with the between-groups factor Hearing status (NH vs. HL), and the two within-groups factors System information (visual vs. visual tactile), and Complexity (lower vs. higher) was used. Main effects of Hearing status, System information and Complexity as well as interaction effects (Hearing status $\times$ System information, Hearing status $\times$ Complexity, System information $\times$ Complexity and Hearing status $\times$ System information $\times$ Complexity) were examined for all performance indicators and measures were these were available.

\section{Participants}

Thirty-two participants (16 with normal hearing; 16 with hearing loss) were recruited from the region around Linköping with help from an Audiology clinic. An inclusion criterion for the hearing loss group (HL) was a moderate hearing loss (41-70 $\mathrm{dB}$ HL) according to WHO categories (Arlinger, 2007) measured with a pure tone average of four mean values, PTA4 (mean of 500, 1000, 2000 and $4000 \mathrm{~Hz}$ ). See Figure 1 for average audiogram of HL participants.

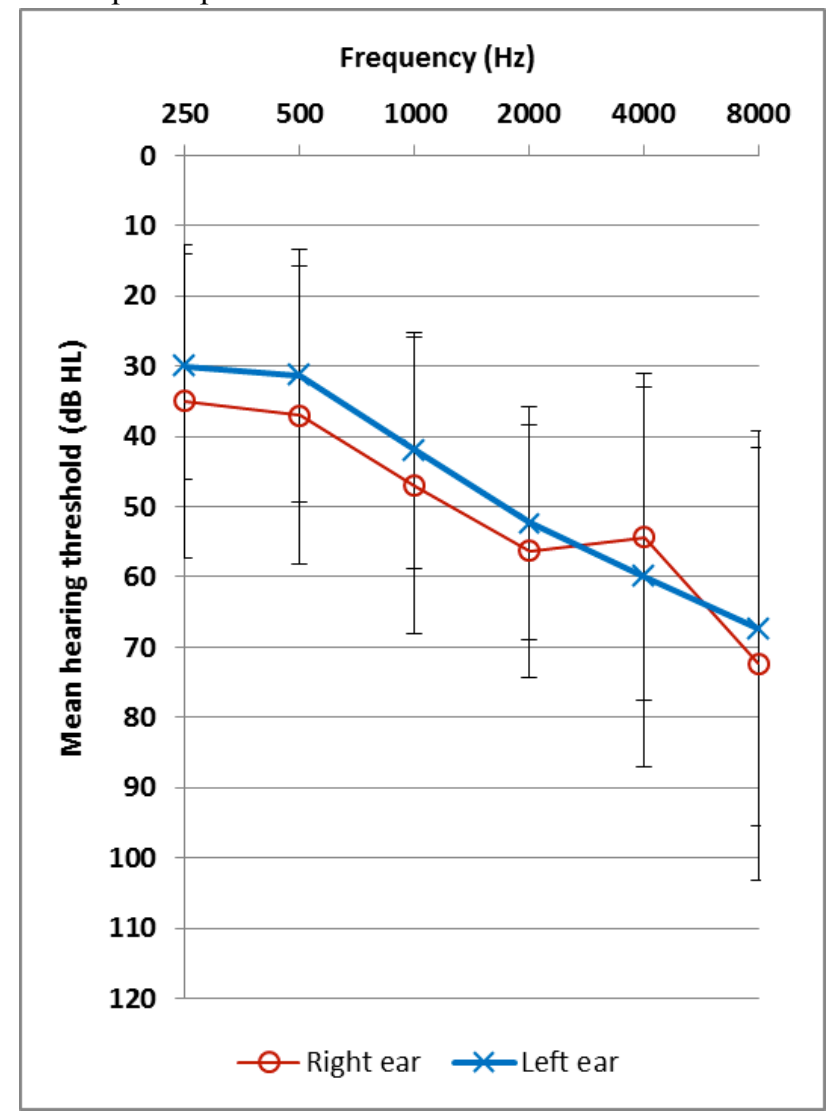

Figure 1: Average audiogram of $H L$ participants
For the normal hearing group (NH) an inclusion criterion was a hearing threshold of maximum $20 \mathrm{~dB} \mathrm{HL}$ at each frequency (500, 1000, 2000 and $4000 \mathrm{~Hz}$ ). Initial hearing screening led to exclusion of one man in $\mathrm{NH}$ who had a hearing loss in one ear and one woman in HL who had only mild hearing loss in one ear. After this, the $\mathrm{NH}$ group included 5 men and 10 women and the HL group included 7 men and 8 women $(N=30 ; n=15$ in the NH group, $n=15$ in the HL group). On average the $\mathrm{NH}$ group drove $1991 \mathrm{~km} /$ year $(S D=1100 \mathrm{~km} /$ year $)$ and the HL group drove $1435 \mathrm{~km} /$ year $(S D=652 \mathrm{~km} /$ year $)$. The mean age was 52.5 years $(S D=10.3$ years $)$ in the $\mathrm{NH}$ group and 56.4 years $(S D=12.4$ years $)$ in the HL group. On average the NH group had had their driving license for 32.2 years ( $S D=10.2$ years) and the HL group for 36.3 years ( $S D=12.4$ years). There was no significant difference between groups in terms of mileage per year, age or years with a driving license.

\section{Procedure}

Upon arrival all participants in the $\mathrm{NH}$ group were screened for hearing loss using a pure tone audiometer and a threshold of maximum $20 \mathrm{~dB}$ HL for PTA4. For participants in the HL group with no audiogram available a complete pure tone audiogram was performed from which a PTA4 was calculated.

All participants completed the following cognitive tests to control for differences between groups; Verbal ability (The F-test, Psykologiförlaget, Stockholm), KIPS test battery including Physical matching, Physical lexical matching, Rhyme and Reading span (Hällgren, Larsby, Lyxell, \& Arlinger, 2001) and a computerized dynamic Trail making test (TMT) (Lehtonen, Dahlström, Hiltunen, \& Summala, 2012). Clinical vision measures included binocular Distance visual acuity using a logMAR chart (Ferris, Kassoff, Bresnick, \& Bailey, 1982) and binocular Contrast sensitivity (log CS) using the Pelli-Robson chart (Pelli, Robson, \& Wilkins, 1988). Both vision tests used letter by letter scoring and a termination rule of 4 out of 5 letters named incorrectly.

Participants were asked to complete a computer-based version of the Useful Field of View test (UFOV®). Skills measured by UFOV are thought to be used during driving (Ball \& Owsley, 1993), and the test is thought to be indicative of accident risk in the older demographic (Ball, Owsley, Sloane, Roenker, \& Bruni, 1993; Owsley et al., 1998). It was, therefore, considered that this test would 
Journal of Eye Movement Research $6(5): 1,1-9$
Thorslund, B., Peters, B., Herbert, N., Holmqvist, K., Lidestam, B., Black, A., Lyxell, B. (2013) Hearing loss and a tactile signal in a navigation system: Driving behavior and eye movements provide a measure of differences in driving-related attentional skills between NH and HL. A mouse-response, PCbased version of UFOV (v 6.1.1, Visual Awareness Research Group Inc.) was used (for an overview of this test see Edwards et al., 2005), and participants were required to perform all three subtests which measure stimulus identification, divided attention, and selective attention respectively. Scores of between 17 and $500 \mathrm{~ms}$ were derived for each subtest by the software using a double staircase presentation method.

All participants undertook two drives of $14 \mathrm{~km}$ each while they performed two preprogrammed navigation tasks guiding them around two different routes in the city of Linköping in Sweden. The same navigation system was used for both drives but during one drive the navigation system presented only the visual information and during the other drive there was an additional vibration in the seat to guide the driver in the right direction. This tactile support was given in either the left or the right side of the driving seat, to indicate the direction of the next turn, and initiated manually at the same position for each participant by pressing one of two buttons connected to the driving seat. The order in which the two routes where driven as well as the order of system information presented was balanced between the groups. To create two levels of workload both routes included two levels of driving complexity, with the lower level comprising driving on a ring road with a speed limit of $70 \mathrm{~km} / \mathrm{h}$, and the higher level driving in the city center with a speed limit of $50 \mathrm{~km} / \mathrm{h}$. The order in which the complexity level was experienced was also balanced between the groups by varying the direction of the route.

\section{Performance indicators and measures}

A VBOX with accompanying software from Racelogic (Racelogic, 2013) was used to measure speed during all conditions. The route was simultaneously recorded with a video camera.

Observations of driving behavior and driving performance assessment were performed with an on-road protocol from Mobility Center in Sweden (Selander, 2011). A matrix covering behaviors regarding maneuver, attention, position, speed adjustment, interaction and planning in situations including roundabouts, crossings, traffic lights, straights, speed bumps and other were filled in with markings of deviations (1 point) and rule breaks (2 points) respectively. The assessment was performed during both drives, however it was not divided into higher and lower driving complexity. Two validations were performed on the On-road protocols. In the first validation, a comparison between the scores from two assessors watching the same ten sample video clips (blinded for hearing status and system information) was made. The first assessor had also done the in car scorings. Intra-class correlations were performed to test the inter rater reliability on four different categories between the two assessors' video clips scores as well as inter rater reliability between the first assessors video clip scores and in car scores respectively, see

Table 1. The correlations between the video clip scores were higher than between the video clip versus incar scores. All correlations were significant except video versus in car for interaction. In the second, markings of speed adjustment were validated by ordering the drives by most speeding's and most "too slow" respectively and comparing with the VBOX data. The ten drives with the largest number of speeding occurrences included seven of the drives with highest average speed. The ten drives with the most "too slow" included six of the drives with lowest average speed.

Table 1: Intra class correlation between two assessors' video clips scores and one assessors video clip scores and in car scores respectively

\begin{tabular}{lcccc}
\hline & Attention & Position & Interaction & Planning \\
\hline $\begin{array}{l}\text { A1 vs. A2 } \\
\text { Video clips }\end{array}$ & $0.84^{*}$ & $0.83^{*}$ & $0.89^{*}$ & $0.91^{*}$ \\
A1 in car & $0.69^{*}$ & $0.81^{*}$ & 0.46 & $0.82^{*}$ \\
$\begin{array}{l}\text { vs. A1 } \\
\text { video clips }\end{array}$ & & & & \\
\hline
\end{tabular}

Eye tracking was recorded during all conditions with SMI ETG glasses (SensoMotoricInstruments, 2013). The field of view was recorded at $1280 \times 960$ pixels and a rate of $30 \mathrm{~Hz}$, with a superimposed gaze marker that shows participant line of gaze. Recording was binocular with built-in parallax compensation, so that accuracy remained good despite varying gaze distances. An IR filter on the glasses compensated for variation in sunlight.

A questionnaire was filled in by the participants after the two drives with the aim to gather the drivers' subjective experience of their driving performance and of the 
Journal of Eye Movement Research $6(5): 1,1-9$
Thorslund, B., Peters, B., Herbert, N., Holmqvist, K., Lidestam, B., Black, A., Lyxell, B. (2013) Hearing loss and a tactile signal in a navigation system: Driving behavior and eye movements information presented by the system in terms of the feelings of safety, usefulness and comfort.

\section{Analysis}

Frame-by-frame analysis, using an assessor blinded to the groups and task, was performed using Interact Software (MangoldInternational, 2013) with predefined regions of interest, see Table 3. From this assessment, Percentage gaze data, Duration of glances and Glances per minute were saved for each region and for each participant respectively for further statistical analysis.

Table 3: Regions of interest used in the gaze data analysis

\begin{tabular}{l}
\hline Regions of interest \\
\hline Windscreen \\
Speedometer \\
Navigation system \\
Rear view mirror \\
Left window + mirror \\
Right window + mirror \\
Other \\
\hline
\end{tabular}

General estimating equations (GEEs) were used to model correlated data from this repeated measures design for the following linear or continuous outcome measures: Speed, On-Road Performance, Gaze behavior patterns and Usability questions. Predictor variables were Tactile (within subjects), Hearing category and age (between subjects). The working correlation matrix was set to exchangeable since symmetry was assumed. Outputs were Wald statistics $(\chi 2)$ showing the significance and a regression coefficient $(B)$ presenting the relation between the groups.

For background questions in the Questionnaire and cognitive tests and vision tests one way ANOVAs were performed.

\section{Results}

\section{Pretests}

There were no significant differences between $\mathrm{NH}$ and HL on any of the cognitive pretest measures of KIPS test battery and neither was there any age effect, see Table 4. The results were in line with previous studies (Hällgren, et al., 2001; Lyxell \& Rönnberg, 1991). Neither was there any significant difference between the groups on the dynamic TMT.
Table 4: Cognitive pretest measures.

\begin{tabular}{llll}
\hline & \multicolumn{3}{c}{ Hearing Status } \\
\cline { 2 - 4 } Correct answers (\%) & $\mathrm{NH}$ & $\mathrm{HL}$ & $p$ \\
\hline Verbal ability & $63.1(7.2)$ & $57.8(10.1)$ & 0.11 \\
Physical matching & $90.1(14.4)$ & $91.0(10.9)$ & 0.85 \\
$\begin{array}{l}\text { Physical lexical } \\
\text { matching }\end{array}$ & $97.9(2.3)$ & $97.8(3.5)$ & 0.94 \\
$\begin{array}{l}\text { Rhyme } \\
\text { Reading span }\end{array}$ & $69.9(11.0)$ & $86.4(11.8)$ & 0.41 \\
& & & \\
& & $56.2(9.0)$ & $5.0)$
\end{tabular}

Neither of the vision tests showed a significant difference between the groups nor was there any self-reported eye conditions. Mean of Distance visual acuity (logMAR) was $-0.14(S D=0.08)$ for $\mathrm{NH}$ and $-0.10(S D=0.09)$ for HL.

There were no significant differences found between $\mathrm{NH}$ and HL on any of the three subtests of UFOV, see Figure 2. However, for the measure of selective attention, a poorer mean score was noted for the HL group, although the mean scores for both groups were well within the normal ranges specified by documentation for the software.

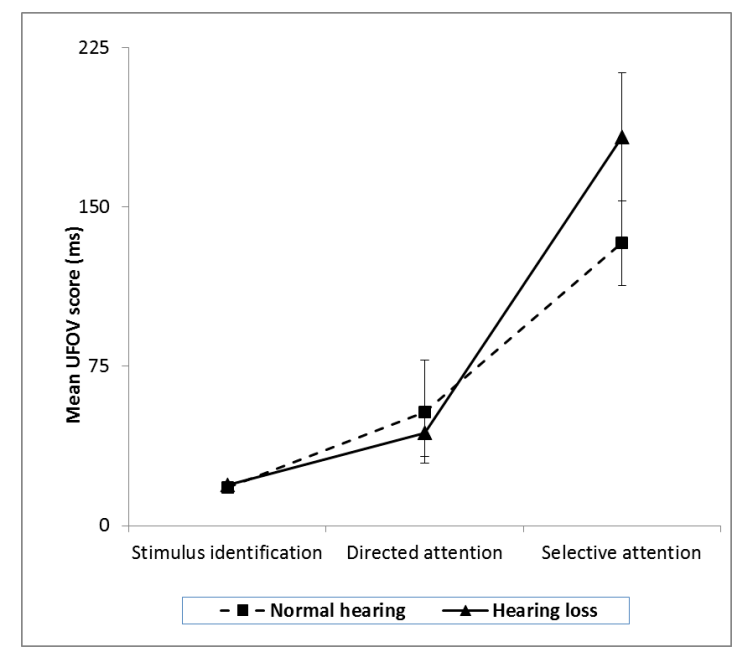

Figure 2: UFOV mean score and standard error mean for $\mathrm{NH}$ and $H L$ respectively 
Journal of Eye Movement Research $6(5): 1,1-9$
Thorslund, B., Peters, B., Herbert, N., Holmqvist, K., Lidestam, B., Black, A., Lyxell, B. (2013) Hearing loss and a tactile signal in a navigation system: Driving behavior and eye movements

\section{Speed}

On road sections with a speed limit of $70 \mathrm{~km} / \mathrm{h}$, the lower driving complexity condition, participants with a hearing loss drove $4 \mathrm{~km} /$ hour slower $(\chi 2=7.06, p<$ $0.05, B=-4.17)$ compared to participants with normal hearing. The same tendency could be seen also on sections with a speed limit of $50 \mathrm{~km} / \mathrm{h}$, that is higher driving complexity; however this was not significant, see Figure 3 . There was no effect of route or navigation system information on the speed.

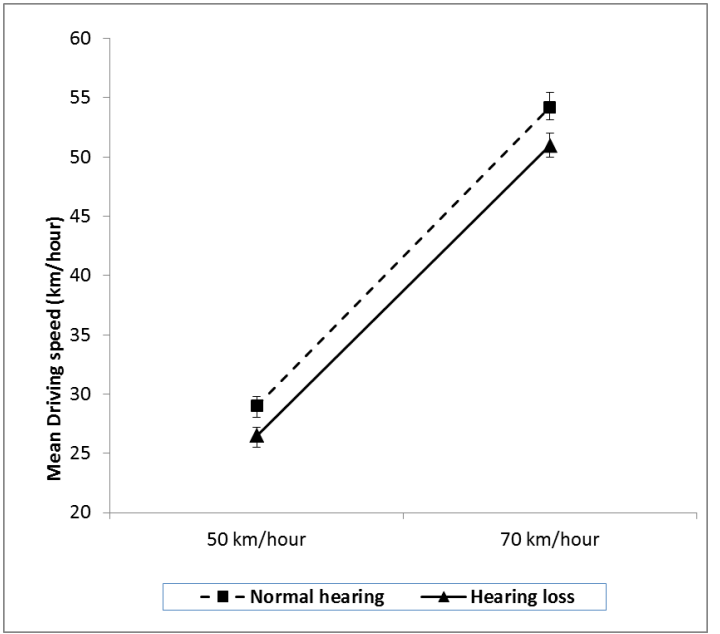

Figure 3: Mean driving speed and standard error mean for both hearing groups at speed limit $50 \mathrm{~km} / \mathrm{h}$ and $70 \mathrm{~km} / \mathrm{h}$ respectively

\section{On-road performance}

When driving without the tactile information active, participants had on average 0.5 more markings on the measure 'Inattention straight' $(\chi 2=9.97, p<0.05, B=$ 0.53 ) and also on average 0.5 more markings on the measure 'Position distance' compared to with the tactile information $(\chi 2=5.81, p<0.05, B=0.53)$. No effect of hearing status was found for system information.

$\mathrm{NH}$ participants had on average 0.3 more markings on the measure 'Speed too high' compared to HL participants $(\chi 2=3.56, p<0.05, B=0.29)$, whereas participants with hearing loss had 0.5 more markings on the measure 'Speed too low' compared to normal hearing participants $(\chi 2=7.02, p<0.05, B=0.46)$. Furthermore participants with hearing loss had on average one more marking on the measure 'uneven speed' compared to normal hearing participants $(\chi 2=7.39, p<0.05, B=1.02)$. No interaction effect between hearing status and gender was found and neither was there an effect of age on any of the on road protocol measures.

\section{Gaze behavior patterns}

With the tactile information active, participants looked on average $7 \%$ less at the navigation display $(\chi 2=$ 17.03, $p<0.05, B=-7.08)$ and consequently on average $7 \%$ more through the windscreen $(\chi 2=11.51, p<0.05, B$ $=7.50$ ), compared to without the tactile information. Looking at the number of glances per minute revealed that without the tactile information, on average participants looked once more per minute at the navigation display $\left(\chi^{2}=23.10, p<0.05, B=1.00\right)$ and there was no effect on the duration of glances. No effect of hearing status appeared on these measures.

HL participants looked on average $1.4 \%$ more in the rear view mirror compared to normal hearing participants $(\chi 2=7.14, p<0.05, B=1.37)$. On average HL participants looked three times as often ( 0.3 compared to 0.1 times per minute) in the rear view mirror $(\chi 2=24.54, p<$ $0.05, B=3.03$ ) compared to the $\mathrm{NH}$ group but there was no effect on the duration of glances. There was no effect of navigation system information on these measures.

No effect of hearing status or of system information was found on gaze data on the speedometer or to the left and to the right. No interaction effect between hearing status and gender was found and neither was there an effect of age on any of the eye tracking measures.

\section{Questionnaire}

With the tactile information active, both hearing groups were significantly more satisfied with the ability to navigate $(\chi 2=31.69, p<0.05, B=1.13)$ and with the help they got from the system $(\chi 2=50.70, p<0.05, B=$ 1.13). Participants also felt higher security $(\chi 2=45.27, p$ $<0.05, B=1.00)$, safety $(\chi 2=34.47, p<0.05, B=0.87)$ and comfort $(\chi 2=14.82, p<0.05, B=0.93)$ in this condition. Furthermore, participants in the hearing loss group were significantly more satisfied compared to the normal hearing group with the ability to navigate when the tactile information was activated $(\chi 2=5.84, p<0.05, B=0.58)$.

There was no significant difference associated with hearing status on self-rated driving performance, mileage per year, frequency of driving in city traffic, having a navigation system in private car, or on any questions regarding the experience of the navigation system when the 
tactile information was inactivated. No age effect was found for the questionnaire results.

\section{Discussion}

The purpose of the present study was to further examine coping strategies associated with hearing loss, evaluate the effect of additional tactile support in a navigation system and to further investigate the effect of hearing loss on eye movement patterns for drivers. We expected tactile support to be associated with more focus on the road, this was suggested by the gaze data showing a decrease in focus on the navigation display when tactile support was activated. In turn this led to increase in the amount of time participants spent looking through the windscreen. The number of glances at the navigation display decreased with tactile support activated, while the duration of these glances was unaffected.

We also expected tactile support to lead to better driving performance, something which was supported by fewer markings on a subjective evaluation of driving performance under the categories 'inattention straight' and 'position distance' with tactile support activated. Also in line with the assumptions were the results of usability questions asked in this study, which showed a higher satisfaction with the navigation system whilst tactile information was activated.

A more cautious driving behavior was expected in the hearing loss group compared to the normal hearing group, specifically it was assumed that hearing impaired drivers would travel at lower speeds based on the findings of previous work (c.f. Picard et al. 2008 and Thorslund et al. 2012). Indeed, this was supported by the data collected, which showed a significantly lower driving speed associated with hearing loss whilst travelling on higher speed limit roads $(70 \mathrm{~km} / \mathrm{h})$ and a similar pattern also appearing whilst on lower speed limit roads (50 $\mathrm{km} / \mathrm{h}$ ). It is reasonable to believe that there are smaller differences in speed at lower speed limits. The results from the On-road protocol were also in line with the speed assumptions, showing less speeding and more occasions of driving too slow for the hearing loss group. Additionally this group had more markings on uneven speed, which could be due to the relationship between sound and the perception of travelling speed (Ohta \& Komatsu, 1991), which may interact with hearing loss in some way. However, the relationship is likely to be very complex and rely on many factors associated with hearing loss beyond sound simply being quieter (i.e. differences in frequency, the onset of the hearing loss, whether hearing aids are being worn). Since sound informs our awareness of the environment whilst driving; it is also possible that HL drivers might, therefore, have less situational awareness whilst driving and move slower to compensate. Perhaps the difficulty in maintaining a consistent speed could also be due to the amount of workload this group is under. Having to pay more attention to sound information used for driving might leave less cognitive resources for driving.

In line with indications from a previous simulator study by Thorslund et al. (2013), it was expected that the hearing loss group would show a tendency to have more glances in the mirrors. This was supported by the gaze data in this study, which showed an association between hearing loss and the number of glances in the rear view mirror. Due to difficulties experienced in distinguishing the left mirror from the left window and the right mirror from the right window, these were clustered respectively and no significant difference was noted for hearing status.

\section{Conclusions}

Results from this study revealed that tactile support leads to higher satisfaction with the navigation system, less time spent to look at the navigation display (in terms of frequency), and thus more focus on road and better driving performance (regarding attention and distance).

The comparison between HL and NH drivers suggests that HL drivers drive slower and look more in the rear view mirror (in terms of frequency). This more cautious driving behavior can be compensatory strategies.

In this study, eye movement behavior is; bound to task (system information), functional in adjusting to the situation and providing necessary visual information to the driver, and can be a part of a compensatory strategy for hearing loss.

\section{References}

Arlinger, S. (Ed.). (2007). Nordisk Lärobok i Audiologi. Nordic Textbook of Audiology. Bromma: C-A Tegnér AB. 
Journal of Eye Movement Research $6(5): 1,1-9$
Thorslund, B., Peters, B., Herbert, N., Holmqvist, K., Lidestam, B., Black, A., Lyxell, B. (2013) Hearing loss and a tactile signal in a navigation system: Driving behavior and eye movements
Ball, K., \& Owsley, C. (1993). The Useful Field of View Test: a new technique for evaluating age-related declines in visual function. Journal of the American Optometric Association, 64(1), 71-79.

Ball, K., Owsley, C., Sloane, M. E., Roenker, D. L., \& Bruni, J. R. (1993). Visual-attention problems as a predictor of vehicle crashes in older drivers. Investigative Ophthalmology \& Visual Science, 34(11), 3110-3123.

Barreto, S. M., Swerdlow, A. J., Smith, P. G., \& Higgins, C. D. (1997). A nested case-control study of fatal work related injuries among Brazilian steel workers. Occupational Environmental Medicine, 54(8), 599-604.

Edwards, J. D., Vance, D. E., Wadley, V. G., Cissell, G. M., Roenker, D., \& Ball, K. K. (2005). Reliability and validity of useful field of view test scores as administered by personal computer. Journal of Clinical and Experimental Neuropsychology, 27(5), 529-543.

Evans, L. (1970). Speed Estimation from a Moving Automobile. Ergonomics, 13(2).

Ferris, F. L., Kassoff, A., Bresnick, G. H., \& Bailey, I. (1982). New visual acuity charts for clinical research. American Journal of Ophthalmol., 94, 91-96.

Fuller, R. (2005). Towards a general theory of driver behaviour. Accidents Analysis and Prevention, 37, 461-472.

Green, K. A., McGwin, G., Owsley, C. ( 2013). Associations Between Visual, Hearing, and Dual Sensory Impairments and History of Motor Vehicle Collision Involvement of Older Drivers. Journal of the American Geriatrics Society., 61(2).

Hickson, L., Wood, J., Chaparro, A., Lacherez, P., \& Marszalek, R. (2010). Hearing Impairment Affects Older People's Ability to Drive in the Presence of Distracters. Journal of the American Geriatrics Society, 58(6), 1097-1103.

Hällgren, M., Larsby, B., Lyxell, B., \& Arlinger, S. (2001). Evaluation of a cognitive test battery in young and elderly normal-hearing and hearingimpaired persons. Journal of the American Academic Audiology, 12(7), 357-370.

Ivers, R., Q., Mitchell, P., \& Cumming, R. (1999). Sensory Impairment and Driving: The Blue Mountains Eye Study. American Journal of Public Health, 89(1), 85-87.

Lehtonen, E., Dahlström, I., Hiltunen, H., \& Summala, H. (2012). On-road visual search and executive functions in elderly drivers. Paper presented at the ICTTP.
Lewis-Evans, B., de Waard, D., Brookhuis, K. (2011). Speed Maintenance under Cognitive Load Implications for Theories of Driver Behaviour. Accidents Analysis and Prevention, 43, 14971507.

Lyxell, B., \& Rönnberg, J. (1991). Visual speech processing: Word-decoding and worddiscrimination related to sentence-based speechreading and hearing-impairment Scandinavian Journal of Psychology, 32(1), 917.

MangoldInternational. (2013). from http://www.mangoldinternational.com

McCloskey, L. W., Koepsell, T. D., Wolf, M. E., \& Buchner, D. M. (1994). Motor-Vehicle Collision Injuries and Sensory Impairments of Older Drivers. [Article]. Age and Ageing, 23(4), 267273.

Ohta, H., \& Komatsu, H. (1991). Speed perception in driving Vision in Vehicles (Vol. III, pp. 415426). Amsterdam: Elsevier Science Publishers.

Owsley, C., Ball, K., McGwin, G., Sloane, M. E., Roenker, D. L., White, M. F., et al. (1998). Visual processing impairment and risk of motor vehicle crash among older adults. Jama-Journal of the American Medical Association, 279(14), 1083-1088.

Pelli, D. G., Robson, J. G., \& Wilkins, A. J. (1988). The design of a new letter chart for measuring contrast sensitivity. Clinical Vision Sciences, 2, 187-199.

Picard, M., Girard, S. A., Courteau, M., Leroux, T., Larocque, R., Turcotte, F., et al. (2008). Could driving safety be compromised by noise exposure at work and noise-induced hearing loss? Traffic Injury Prevention, 9(5), 489-499.

Racelogic. (2013). from http://www.racelogic.co.uk/

Roth, T. N., Hanebuth, D., Probst, R. (2001). Prevalence of age-related hearing loss in Europe: a review. European Archives of Otorhinolaryngol, 268(8), 1101-1107.

Selander, H., Lee, H.C., Johansson, K., Falkmer, T. (2011). Older drivers: On-road and off-road test results. Accidents Analysis and Prevention., 43.

SensoMotoricInstruments. (2013). from http://www.eyetracking-glasses.com

Thorslund, B., Ahlstrom, C., Peters, B., Eriksson, O., Lyxell, B., \& Lidestam, B. (2013). Cognitive workload and visual behavior in elderly persons with hearing loss. (In submission). 
Thorslund, B., Peters, B., Lidestam, B., \& Lyxell, B. (2013). Cognitive workload and driving behavior in persons with hearing loss.

Transportation Research Part F: Psychology and Behaviour 21C, 113-121.

Thorslund, B., Peters, B., Lyxell, B., \& Lidestam, B. (2013). The Influence of Hearing Loss on Transport Safety and Mobility. European Transport Research Review, 5(3), 117-127. 